\title{
Are hypertrophic astrocytes a sufficient criterion of perinatal telencephalic leucoencephalopathy? ${ }^{1}$
}

\author{
ALAN LEVITON AND FLOYD H. GILLES \\ From the Departments of Neurology and Pathology (Neuropathology), \\ The Children's Hospital Medical Center, and the Department of Neurology-Neuropathology, \\ Harvard Medical School, Boston, Massachusetts 02115, U.S.A.
}

SUMMARY To determine if the presence of amphophilic globules (GL) in infant cerebral white matter was a necessary criterion of perinatal telencephalic leucoencephalopathy (PTL), the epidemiological features of infants who had PTL - that is, hypertrophic astrocytes and amphophilic globules (HA.GL) -in their cerebral white matter were compared with those of infants who had hypertrophic astrocytes, but who did not have amphophilic globules (HA. $\overline{\mathrm{GL}})$. Postmortem bacteraemia was seen much more frequently in infants with HA.GL than in infants with HA. $\overline{\mathrm{GL}}$ $(P<0.05)$. In addition, infants with $\mathrm{HA} \cdot \mathrm{GL}$ tended to die at older postnatal ages than infants with $\mathrm{HA} \cdot \overline{\mathrm{GL}}$. These observations are in keeping with the view that HA.GL and HA. $\overline{\mathrm{GL}}$ are not epidemiologically identical. The operational definition of PTL therefore remains the occurrence of both HA and GL in infant cerebral white matter.

Focal necrosis of cerebral white matter in infants has been appreciated as an abnormal finding since the time of Virchow (1868). Only in recent years, however, has there been appreciation of other morphological abnor.nalities of infant cerebral white matter (Gilles and Murphy, 1969).

In preparation for epidemiological studies designed to evaluate potential 'causes' of these abnormalities, it was necessary to determine if each abnormality was independent of the others. This was achieved by a search for clusters (Leviton and Gilles, 1971). ${ }^{2}$ Hypertrophic astrocytes (HA) and perivascular amphophilic globules (GL) occurred together much more frequently than was expected if these were independent variables. Because of this strong association of HA with GL, it was considered

\footnotetext{
1 This study was supported in part by funds provided by the United Cerebral Palsy Research and Educational Foundation (R-244-69), by the Program-Project Grant No. NSI-EP, 1 P01 NS 09704-01 NSPA, HD, NINDS, and The Children's Hospital Medical Center Mental Retardation and Human Development Research Program (HD 03-0773), NICHD.

2 Abbreviations: HA: hypertrophic astrocytes, GL: amphophilic globules, PTL: perinatal telencephalic leucoencephalopathy, $\mathbf{H A} \cdot \overline{\mathbf{G L}}$ : hypertrophic astrocytes in the absence of amphophilic globules, HA.GL: hypertrophic astrocytes with amphophilic globules, $\overline{\mathbf{H A}}$ : the absence of hypertrophic astrocytes, PMB: postmortem bacteraemia.
}

that their occurrence together in the cerebral white matter of infants might represent a distinct morphological entity. The name perinatal telencephalic leucoencephalopathy (PTL) was applied to this entity.

In a number of older children and adults we have seen small firm brains with a prominent reduction of cerebral white matter in all regions except the anterior temporal lobes (Gilles, Leviton, and Murphy). The clinical history of these patients and the morphology of their brains appeared compatible with predictions of the sequelae of PTL (Gilles and Murphy, 1969). Firstly, the paucity of white matter is in keeping with the idea of interference with the process of myelinogenesis. Secondly, the fibrillary gliosis is compatible with the prior presence of HA. Thirdly, the lack of anterior temporal lobe involvement in the face of gliosis and paucity of white matter elsewhere in the cerebral hemispheres is what may be expected with an insult occurring before the time of myelinogenesis in the anterior temporal lobe-that is, before the middle of the first year.

Two hypotheses have been formulated to account for both the paucity of white matter and 
the white matter gliosis. In one of these hypotheses, an insult to myelinogenesis results in the release of substances that serve as a stimulus for the presence of HA. In the other hypothesis, an insult to myelinogenesis results in the transformation of pre-myelin glial cells to HA. In either of these hypotheses the presumed late sequelae of PTL are adequately accounted for by the prior presence of HA. There is no need to involve the prior presence of GL. The possibility was therefore considered that HA in the cerebral white matter of young infants, with or without GL, might be an adequate criterion of PTL.

Our understanding of cerebral white matter abnormalities in infants has been facilitated by employing concepts of set theory (Leviton and Gilles, 1971). The set of HA, which consists of all infants who died with HA in their cerebral white matter, may be divided into subsets. We are concerned here with only two subsets of HA: $\mathrm{HA} \cdot \overline{\mathrm{GL}}$ (HA without GL) and HA.GL (HA with GL). The entity of PTL has been operationally defined (vide infra) as the subset HA.GL. To extend the definition of PTL to the entire set of HA, the subset HA. $\overline{\mathrm{GL}}$ should have epidemiological features virtually identical with those of the subset HA.GL. Thus, anencephaly and spina bifida have been considered 'as one causal entity rather than two' (MacMahon and Pugh, 1970), because of similarities of association with ethnic groups, socioeconomic status, sex, maternal age and parity, similarity in trends of frequency over time, and the occurrence of both anomalies in the same family (MacMahon, Pugh, and Ingalls, 1953).

If the entity $\mathrm{HA} \cdot \overline{\mathrm{GL}}$ and the entity $\mathrm{HA} \cdot \mathrm{GL}$ have very similar epidemiological features, then the two may be merged to form an entity characterized by HA. This would facilitate future epidemiological studies. The following report is a comparison of the epidemiological features of $\mathrm{HA} \cdot \overline{\mathrm{GL}}$ and of HA.GL.

\section{METHODS}

The base population consisted of all 191 infants who survived birth, died at Children's Hospital Medical Center of Boston between 1 January 1965 and 31 December 1967, with a postnatal age of less than 4 months, were free of gross intracranial abnormalities associated with white matter injury, did not have diseases known to injure the central nervous system, had microscopic examination of the brain, and for whom adequate epidemiological data were available.

The procedure for preparation of material for neuropathological study is detailed elsewhere (Gilles 0 and Murphy, 1969). During a six month period, the slides of all 191 infants were examined separately by each of two neuropathologists who had no knowledge of the clinical history or general necropsy findings. For the purposes of this study, each infant was $\vec{\Rightarrow}$ placed into one of three mutually exclusive and $\frac{\mathcal{O}}{5}$ exhaustive morphological categories: (1) presence of $\frac{\text { 응 }}{6}$ hypertrophic astrocytes and absence of amphophilic $\frac{\bar{O}}{\bar{D}}$ globules (HA. $\overline{\mathrm{GL}})$, (2) presence of hypertrophic $\vec{\varnothing}$ astrocytes and amphophilic globules (HA.GL), and $\bigcirc$ (3) absence of hypertrophic astrocytes $(\overline{\mathrm{HA}})$. All के statistical evaluations were limited to comparing $\vec{O}$ infants with $\mathrm{HA} \cdot \overline{\mathrm{GL}}$ with infants with $\mathrm{HA} \cdot \mathrm{GL}$.

Necropsy records were reviewed without knowledge of the white matter histopathology. Anamnestic $\overline{\vec{z}}$ and anatomical information about potential risk흥 factors was obtained from each necropsy record. The anamnestic information consisted of: (1) gest羿 $\omega$ tional age, (2) date of death, (3) postnatal age foc $_{\infty}$ death, (4) whether or not there was mention of ${ }^{\infty}$ cardiac catheterization, thoracic surgery, or a俩-을 dominal surgery, (5) whether or not an organisinwas cultured from blood aspirated from the heart ot the time of postmortem examination.

Those risk factors of an anatomical nature we morphological evidence of congenital heart diseases, $\omega$ hyaline membrane disease, amniotic fluid aspiration, $\square$ pneumonia, and peritonitis.

An infant was considered preterm when the duration of gestation was less than 36 weeks by historyg (43 infants). When the duration of gestation was not这 known, the criteria for classifying an infant as preterm were crown-heel length less than $45 \mathrm{~cm}$ 응 (Gruenwald and Minh, 1960) and histological evidence of glomerulogenesis (Kissane and Smith, 1967) (eight infants).

Postnatal age at death may be an indirect measure of when an insult could have occurred, whereas total? (gestational plus postnatal) age at death may be a measure of 'vulnerability' or 'responsiveness' of maturing cerebral white matter (Leviton and Gilles, $\frac{3}{3}$ 1973b). The population of infants with HA in their cerebral white matter, therefore, was classified by postnatal age. The four postnatal age groupings were those that divided the base population into approximate quarters (Leviton and Gilles, 1973a) N The five total age groupings were: $<36$ weeks, 37-39 weeks, 40 weeks, $41-43$ weeks, and $>43$ weeks.

Three null hypotheses were tested. The basic formw of the first null hypothesis is that the cumulative 
frequency of deaths by age of infants with $\mathrm{HA} \cdot \overline{\mathrm{GL}}$ does not differ from that of infants with HA.GL. This was tested separately for postnatal age and total (gestational plus postnatal) age. The probability of the random occurrence of the observed differences was determined by the KolmogorovSmirnov two-sample test (Siegel, 1956).

The second null hypothesis is: the distribution of the annual occurrence of $\mathrm{HA} \cdot \overline{\mathrm{GL}}$ during the three years of this study does not differ from the annual occurrence of HA.GL. A $\chi^{2}$ value was obtained for the observed distribution.

The generalized form of the last null hypothesis is: in the population of infants who had HA in their cerebral white matter, the occurrence of GL is independent of each risk factor. This was tested separately for distributions by postnatal age and total age by the Mantel-Haenszel procedure (Mantel and Haenszel, 1959).

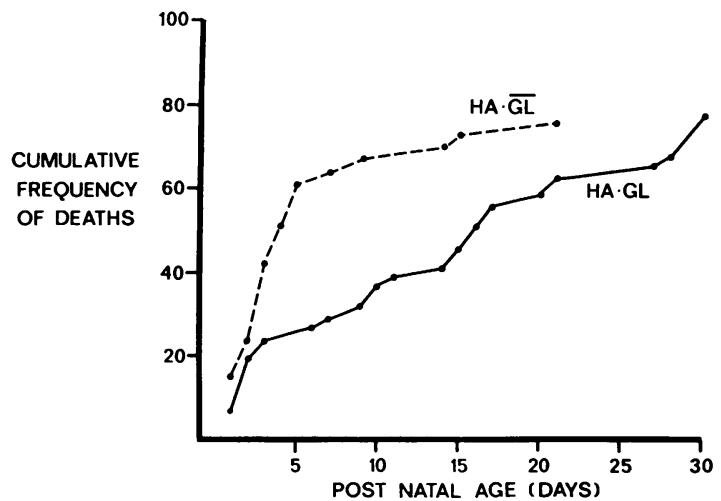

FIG. 1. Infants with $H A \cdot \overline{G L}$ tended to die at appreciably younger postnatal ages than infants with $H A \cdot G L(P<0.05)$.

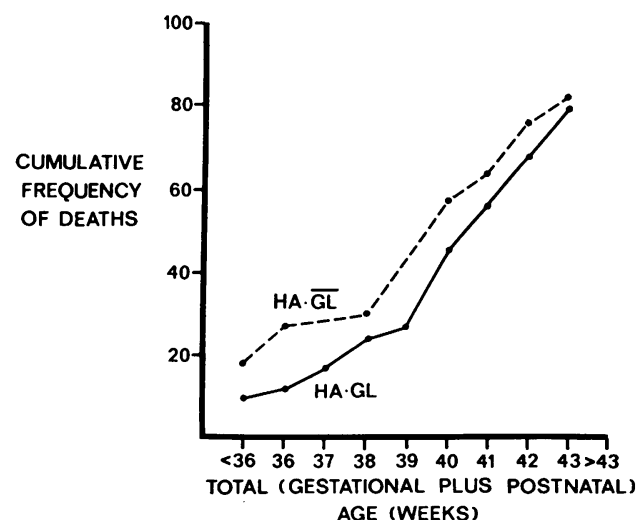

FIG. 2. Infants with $H A \cdot \overline{G L}$ tended to die at minimally younger total ages than infants with $H A \cdot \overline{G L}$.

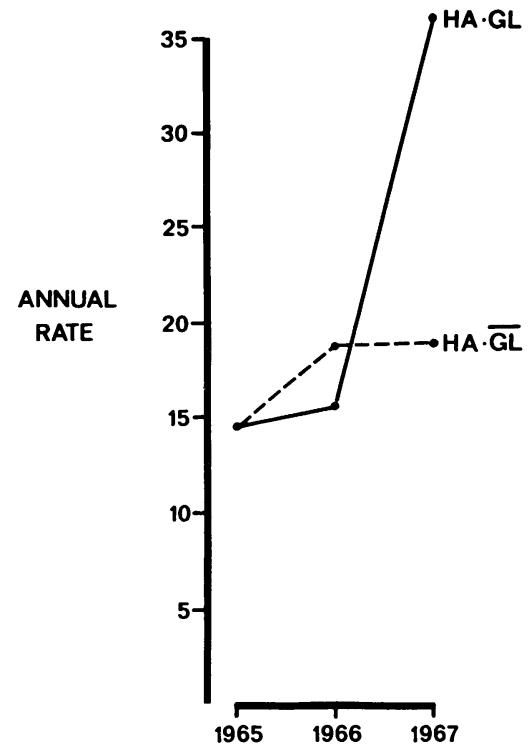

FIG. 3. The marked rise in the frequency of occurrence of $\mathrm{HA} \cdot \mathrm{GL}$ in 1967 is in sharp contrast with the almost imperceptible increase in the frequency of $H A \cdot \overline{G L}$. The observed distribution of all infants with $H A$ by whether or not they had GL and by year of death, however, may represent random phenomena $\left(\chi^{2}=2 \cdot 52,0 \cdot 2<P<0.3\right.$ with 2 degrees of freedom).

\section{RESULTS}

Of the 191 infants in the study population 33 had HA. $\overline{\mathrm{GL}}$ and 41 had HA.GL. Infants with HA.GL tended to die at older postnatal ages than infants with $\mathrm{HA} \cdot \overline{\mathrm{GL}}(\mathrm{P}<0.05$ by the Kolmogorov-Smirnov two-tailed test) (Fig. 1). On the other hand, the tendency for infants with HA.GL to die at older ages than infants with HA.GL is minimal when infants are classified by total (gestational plus postnatal) ages (Fig. 2).

The annual rate of HA. $\overline{\mathrm{GL}}$ was not appreciably different from that of HA. GL in both 1965 and 1966 (Fig. 3). In 1967, however, the rate of $\mathrm{HA} \cdot \mathrm{GL}$ was almost double that of HA. $\overline{\mathrm{GL}}$. The distribution by year of death of infants with $\mathrm{HA} \cdot \overline{\mathrm{GL}}$, nevertheless, is not significantly different from that of infants with HA. GL $\left(\chi^{2}=2 \cdot 52\right.$ with 2 d.f., $0 \cdot 2<\mathrm{P}<0 \cdot 3$ ).

In infants with $\mathrm{HA}$ in their cerebral white matter, GL are positively associated with post- 
TABLE 1

SUMMARY CHI SQUARE VALUES FOR ASSOCIATION OF GL WITH SELECTED RISK FACTORS IN INFANTS WITH HA*

\begin{tabular}{ll}
\hline Risk factor & Summary $\chi^{2}$ \\
\hline Congenital heart disease & $0 \cdot 39$ \\
Postmortem bacteraemia & $4 \cdot 31 \dagger$ \\
Pneumonia & $0 \cdot 00$ \\
Peritonitis & $0 \cdot 13$ \\
Amniotic aspiration & $0 \cdot 09$ \\
Hyaline membranes & $0 \cdot 02$ \\
Laparotomy & $0 \cdot 02$ \\
Thoracotomy & $0 \cdot 08$ \\
Cardiac catheterization & $0 \cdot 11$ \\
Gestational age <36 weeks & $5 \cdot 40 \dagger$
\end{tabular}

* The infants have been classified by postnatal ages for these de terminations. GL are positively associated with postmortem bacteraemia and negatively associated with preterm birth.

$+\mathbf{P}<0.05$.
TABLE 2

SUMMARY CHI SQUARE VALUES FOR ASSOCIATION OF GL WITH SELECTED RISK FACTORS IN INFANTS WITH HA*

\begin{tabular}{lcl}
\hline Risk factor & Summary $\chi^{2}$ \\
Congenital heart disease & 0.34 \\
Postmortem bacteraemia & 4.43 \\
Pneumonia & $0 \cdot 19$ \\
Peritonitis & 1.25 \\
Amniotic aspiration & $0 \cdot 15$ \\
Hyaline membranes & 0.01 \\
Laparotomy & 0.00 \\
Thoracotomy & 0.09 \\
Cardiac catheterization & 2.69 \\
Gestational age <36 weeks & $0 \cdot 18$ & \\
\hline
\end{tabular}

* The infants have been classified by total (gestational plus postnatal) ages for these determinations. GL are positively associated with postmortem bacteraemia.

$+\mathrm{P}<0.05$.

TABLE 3

DISTRIBUTION OF INFANTS WITH HA $\overline{\text { GL }}$ AND HA·GL BY WHETHER OR NOT THEY HAD POSTMORTEM BACTERAEMIA (PMB)

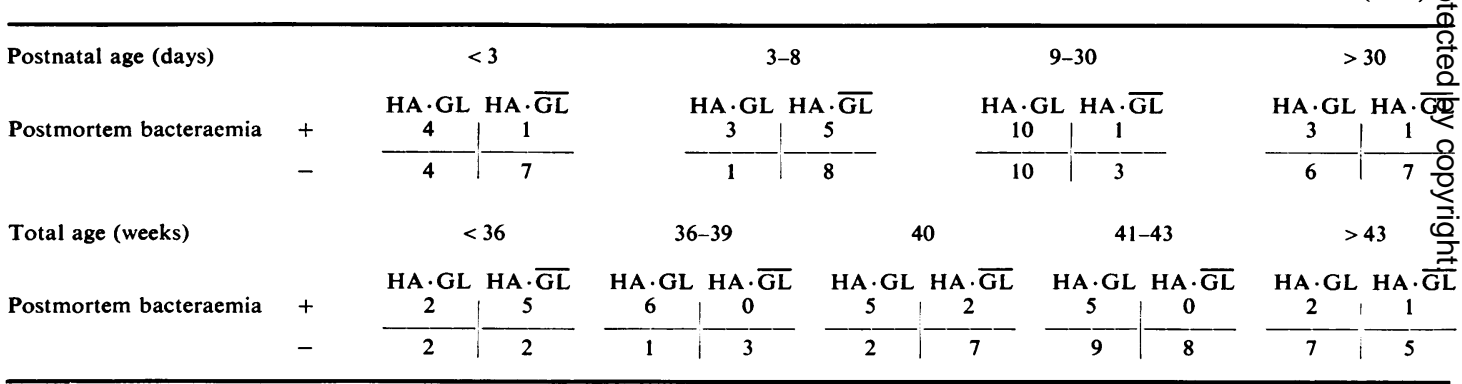

* The infants are classified by postnatal age in the top set of fourfold tables and by total (gestational plus postnatal) age in the lower set.

TABLE 4

DISTRIBUTION OF INFANTS WITH HA $\overline{\mathrm{GL}}$ AND HA $\cdot \mathrm{GL}$ BY WHETHER OR NOT THEY HAD PRETERM BIRTH*

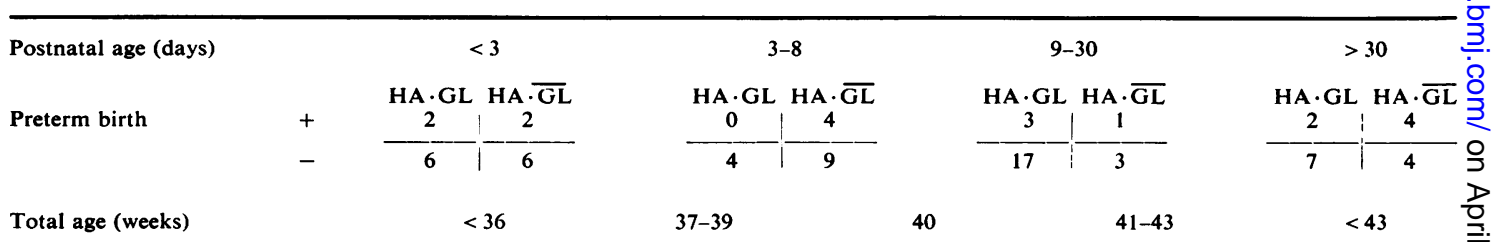

\begin{tabular}{|c|c|c|c|c|c|c|c|c|c|c|c|}
\hline Preterm birth & + & $\underset{4}{\mathrm{HA} \cdot \mathrm{GL}}$ & $\begin{array}{c}\mathbf{H A} \cdot \overline{\mathbf{G L}} \\
7\end{array}$ & $\underset{2}{\mathrm{HA} \cdot \mathrm{GL}}$ & $\underset{1}{\mathrm{HA} \cdot \overline{\mathrm{GL}}}$ & $\begin{array}{c}\mathrm{HA} \cdot \mathrm{GL} \\
0\end{array}$ & $\begin{array}{c}\mathbf{H A} \cdot \overline{\mathrm{GL}} \\
\mathbf{0}\end{array}$ & $\underset{1}{H A \cdot G L}$ & $\underset{2}{H A \cdot \overline{G L}}$ & $\begin{array}{c}\mathrm{HA} \cdot \mathrm{GL} \\
\mathbf{0}\end{array}$ & $\underset{1}{H A \cdot G L}$ \\
\hline & - & 0 & 0 & 5 & 2 & 7 & 9 & 13 & 6 & 9 & 5 \\
\hline
\end{tabular}

* The infants are classified by postnatal age in the top set of fourfold tables and by total (gestational plus postnatal) age in the lower set. 
mortem bacteraemia regardless of whether the infants are classified by postnatal age only or by total age (Tables 1 to 3 ). The negative association of GL with preterm birth, evident when the infants are classified by postnatal age, is virtually nonexistent when infants are classified by total age (Table 4).

\section{DISCUSSION}

Aetiology is best considered as dealing not with the 'causes' of an entity in a narrow sense of that term, but rather with those factors that increase the probability of that entity occurring. It is for this reason that the present study is concerned with 'risk factors'.

An operational definition provides objective criteria by which any observer can decide, for any particular case, whether the term does or does not apply to that case (Bridgeman, 1927). This allows testing of the hypothesis on which the definition is based.

The decision to broaden or narrow an operational definition should be a function of the purpose served by definition. At present, the goal of our studies of infant cerebral white matter abnormalities is the recognition of risk factors. A broadened definition of PTL could minimize the population size necessary to identify a risk factor-that is, it could increase efficiency (Miettinen, 1970). On the other hand, a broader definition could increase bias in the estimation of the effect under study-that is, it might reduce validity (Miettinen, 1970). Any increase in efficiency, however, should not be at the expense of validity.

Differences in the ability of infant cerebral white matter to respond to an insult may be a function of total (gestational plus post-natal) age (Leviton and Gilles, 1973b). Evidence for this 'total age' concept was obtained by evaluating the frequency of selected combinations of morphological abnormalities in infants who had postmortem bacteraemia, a risk factor clearly associated with HA.GL (Leviton and Gilles, 1972). In that population, the risk of $\mathrm{HA} \cdot \overline{\mathrm{GL}}$ occurred at a younger total age than the peak risk of HA. GL. In the data reported here, however, the distribution of infants with $\mathrm{HA} \cdot \overline{\mathrm{GL}}$ by total age, does not differ significantly from that of infants with HA.GL. This lack of appreciable difference between total age specific risks of $\mathrm{HA} \cdot \overline{\mathrm{GL}}$ and HA.GL would therefore appear to reflect the effect not only of bacteraemia but also of other insults. This is compatible with the hypothesis that HA. $\overline{\mathrm{GL}}$ and $\mathrm{HA} \cdot \mathrm{GL}$ are not necessarily aetiologically identical. The prominent difference in postnatal age distributions between HA. $\overline{\mathrm{GL}}$ and HA.GL does not help in distinguishing differences in aetiology for it is compatible with differences in the time of occurrence of a single insult-that is, response is a function of infant's age - or with differences in the time of multiple insults - that is, response is a function of age and/or nature of insult.

The prominent increase in frequency of HA.GL in 1967 quite closely resembled the increased frequency of PMB in the base population in 1967 (Leviton and Gilles, 1973a). The difference in temporal patterns between $\mathrm{HA} \cdot \overline{\mathrm{GL}}$ and HA.GL (Fig. 3) may therefore be additional evidence that the two subsets are not aetiologically identical. The most convincing evidence that $\mathrm{HA} \cdot \overline{\mathrm{GL}}$ and HA.GL are not epidemiologically identical is that $\mathrm{HA} \cdot \mathrm{GL}$ is strongly associated with postmortem bacteraemia, whereas $\mathrm{HA} \cdot \overline{\mathrm{GL}}$ is not (Tables 1 and 2). Since $\mathrm{HA} \cdot \overline{\mathrm{GL}}$ and HA.GL are not epidemiologically identical a loss of validity may be expected if the definition of PTL were broadened to include the entire set of HA. The operational definition of PTL, therefore, will not be broadened.

The authors are indebted to Mrs. Denise Osterberg for manuscript preparation.

\section{REFERENCES}

Bridgeman, P. W. (1927). The Logic of Modern Physics. Macmillan: New York.

Gilles, F. H., Leviton, A., and Murphy, S. F. Morphologic sequelae of perinatal telencephalic leucoencephalopathy. (In preparation.)

Gilles, F. H., and Murphy, S. F. (1969). Perinatal telencephalic leucoencephalopathy. Journal of Neurology, Neurosurgery, and Psychiatry, 32, 404-413.

Gruenwald, P., and Minh, H. N. (1960). Evaluation of body and organ weights in perinatal pathology. American Journal of Clinical Pathology, 34, 247-253.

Kissane, J. M., and Smith, M. G. (1967). Pathology of Infancy and Childhood, p. 519. Mosby: St. Louis.

Leviton, A., and Gilles, F. H. (1971). Clustering of the morphological components of perinatal telencephalic leucoencephalopathy. Journal of Neurology, Neurosurgery, and Psychiatry, 34, 642-645.

Leviton, A., and Gilles, F. H. (1973a). An epidemiologic 
study of perinatal telencephalic leucoencephalopathy in an autopsy population. Journal of Neurological Science. (In press.)

Leviton, A., and Gilles, F. H. (1973b). Morphologic abnormalities in human infant cerebral white matter as a function of gestational and postnatal age. (Submitted for publication.)

MacMahon, B., Pugh, T. F., and Ingalls, T. H. (1953). Anencephalus, spina bifida, and hydrocephalus. Incidence related to sex, race, and season of birth, and incidence in siblings. British Journal of Preventive and Social Medicine, 7, 211-219.
MacMahon, B., and Pugh, T. F. (1970). Epidemiology: Principles and Methods, pp. 53-54. Little, Brown: Boston. Mantel, N., and Haenszel, W. (1959). Statistical aspects of the analysis of data from retrospective studies of disease. Journal of the National Cancer Institute, 22, 719-748.

Miettinen, O. S. (1970). Matching and design efficiency in retrospective studies. American Journal of Epidemiology, 91, 111-118.

Siegel, S. (1956). Nonparametric Statistics for the Behavioral Sciences, pp. 127-136. McGraw-Hill: New York.

Virchow, R. (1868). Ueber interstitielle Encephalitis. Archiv für Pathologische Anatomie, 44, 472-476. 